\title{
The ratio of gastric tube length to thorax length: a vital factor affecting leak after esophageal cervical anastomosis
}

\author{
Xiao-Kun Li ${ }^{1 \#} \wedge$, Tian-Tian Hua ${ }^{2 \#}$, Chi Zhang ${ }^{3 \#}$, Yang Xu ${ }^{4}$, Wen-Jie Wu ${ }^{5}$, Chao Zheng ${ }^{1}$, Gao-Ming Wang ${ }^{3}$, \\ Yong Qiang', Zhuang-Zhuang Cong ${ }^{3}$, Jun Yi, ${ }^{1,3,4}$, Yi Shen ${ }^{1,3,4,5}$ \\ ${ }^{1}$ Department of Cardiothoracic Surgery, Jinling Hospital, School of Medicine, Southeast University, Nanjing, China; ${ }^{2}$ Department of Epidemiology \\ and Biostatistics, School of Public Health, Southeast University, Nanjing, China; ${ }^{3}$ Department of Cardiothoracic Surgery, Jinling Hospital, Medical \\ School of Nanjing University, Nanjing, China; ${ }^{4}$ Department of Cardiothoracic Surgery, Jinling Hospital, Jinling School of Clinical Medicine, \\ Nanjing Medical University, Nanjing, China; ${ }^{5}$ Department of Clinical Medicine, School of Medicine, Southeast University, Nanjing, China \\ Contributions: (I) Conception and design: XK Li, Y Shen; (II) Administrative support: Y Shen, J Yi, ZZ Cong; (III) Provision of study materials or \\ patients: XK Li, Y Shen, J Yi; (IV) Collection and assembly of data: XK Li, TT Hua, C Zhang; (V) Data analysis and interpretation: TT Hua, Y Xu, \\ WJ Wu; (VI) Manuscript writing: All authors; (VII) Final approval of manuscript: All authors. \\ \#These authors contributed equally to this work. \\ Correspondence to: Yi Shen. Department of Cardiothoracic Surgery, Jinling Hospital, School of Medicine, Southeast University, Nanjing, China. \\ Department of Cardiothoracic Surgery, Jinling Hospital, Medical School of Nanjing University, Nanjing, China. Email: dryishen@nju.edu.cn; Jun \\ Yi. Department of Cardiothoracic Surgery, Jinling Hospital, Jinling School of Clinical Medicine, Nanjing Medical University, Nanjing, China. \\ Email: njyijun@163.com; Zhuang-Zhuang Cong. Department of Cardiothoracic Surgery, Jinling Hospital, Medical School of Nanjing University, \\ Nanjing, China. Email: dr.congzz@gmail.cn.
}

Background: Esophagogastric anastomotic leak (AL) is a severe complication following esophageal resection. This study aims to explore preliminarily whether the ratio of the gastric conduit length to the thorax length can be regarded as a potential prognostic variable for AL, and if so, a cut-off value can be found to divide the patients into distinct risk groups.

Methods: We retrospectively reviewed the clinical data of 273 patients who underwent esophagectomy. The gastric conduit length, the thorax length, and other covariates were collected. Logistic regression was first conducted to probe the rationality of the ratio as a risk indicator of AL. Then the dichotomizing analysis was applied to find the optimal cut-off value.

Results: The incidence of AL was 12.5\% (34/273). The coefficient of the ratio in the logistic regression equation was -7.901 with $\mathrm{P}<0.001$, which indicated that the larger the ratio, the smaller the risk of AL. Further smoothed scatter plots revealed that a potential step function of the ratio of AL incidence exists, of which the steep part ranges from 1.74 to 1.90 . Results of the accurate cut-off value search through a minimum $\mathrm{P}$ value approach give the optimal dichotomization point of 1.79 .

Conclusions: The ratio of the gastric conduit length to the thorax length can reflect the tension in the anastomosis. The research proposes that surgeons can control the length of the gastric conduit during reconstruction to reduce the tension in the anastomosis and thus lead to a decrease in the incidence of AL.

Keywords: Esophagectomy; anastomotic leak; ratio of gastric conduit length to thorax length; dichotomization

Submitted Aug 26, 2020. Accepted for publication Dec 21, 2020.

doi: $10.21037 / \mathrm{atm}-20-6082$

View this article at: http://dx.doi.org/10.21037/atm-20-6082

$\wedge$ ORCID: 0000-0002-1432-5573. 


\section{Introduction}

The standard treatment for esophageal carcinoma is esophagectomy. The stomach is most commonly used to reconstruct the alimentary tract for esophageal cancer. Anastomotic leakage (AL) is an important complication following esophageal resection and an important cause of death (1). The incidence of AL ranges from $6.2 \%$ to $27 \%$ (2-6). In the Chinese population, the intrathoracic AL rate is reported to be $2.8 \%$ to $6.6 \%$, whereas the cervical $\mathrm{AL}$ rate is $7.9 \%$ to $24 \%$ (7). AL is also a major cause of perioperative morbidity and mortality. Patients with AL have three times the risk of death as those without $\mathrm{AL}$, and the mortality rate of the former can reach up to $60 \%(8,9)$. Unlike cervical anastomosis, initial presentations of AL may include a neck flare-up and redness at the surgical incision with pus, salivatype fluid, or air discharge from the wound, accompanied by septic signs. Often, the only signs of leakage are increased heart rate, increased body temperature, or bilious drainage from the surgical drain; other times, a leakage is simply radiologically determined without external signs, since it is not uncommon for these leakages to present as mediastinitis without wound discharge. Occasionally, infection can spread to the thorax and generate an infection of the pleural cavity or mediastinitis, if not treated in time (10).

The gastric conduit is commonly used for reconstruction as a substitute with sufficient length after esophageal anastomosis. According to related studies, the risk factors of anastomotic leakage after esophagectomy include older age, ischemia of the gastric conduit, malnutrition, renal insufficiency, diabetes, high body mass index and surgical approach $(5,11,12)$. Three studies considered that poor tissue perfusion is a major cause of anastomotic leakage (13-15). In this study, we aimed to explore the influence of gastric conduit length on AL. The gastric conduit length without tension, reflecting the initial length, was measured before the gastric conduit was pulled up to the neck during surgery, and the thorax length, which starts from the xiphoid and ends at the sternal notch, was measured before surgery. An index that indicated the tension of the gastric conduit was then constructed by dividing the gastric conduit length (i.e., the initial flaccid gastric conduit length minus the redundant portion length) by the thorax length, and we speculated that this tension was a major cause of AL. We present the following article in accordance with the MDAR reporting checklist (available at http://dx.doi.org/10.21037/ atm-20-6082).

\section{Methods}

\section{General description}

We retrospectively reviewed the clinical information of 302 patients undergoing esophagectomy and cervical anastomosis between 2016 and 2020 at the Department of Thoracic Surgery in Nanjing Jinling Hospital. And 29 patients were excluded for lack of the data associated with the length of the gastric conduit length or/and the thorax length. If other data were missing, an imputation method was used to integrate the data. Finally, 273 patients were included in this study. The study was conducted in accordance with the Declaration of Helsinki (as revised in 2013). The study was approved by the Institutional Review Board of the Jinling Hospital (approval number 2018NZKY-027-54). The ethics committee waived the need for informed consents from those patients since our study was a retrospective cohort analysis and analyzed anonymously. The perioperative data, which consisted of demographic indexes, surgically related indexes, tumorrelated indexes, and hematological indexes, were collected and summarized by analyzing the medical records database. The C-reactive protein (CRP) level was measured on postoperative day 3 (POD3) since early AL always occurs at POD3-POD7 and the CRP level at POD1 could mostly influenced by the procedure of the surgery; thus, the CRP level at POD3 could be considered an early predictor of AL. Logistic regression and a dichotomizing approach were applied to obtain an optimal cut-off point.

\section{Definition of $A L$}

AL was defined as follows: (I) leakages confirmed by endoscopy, chest computed tomography (CT), and/ or surgical exploration (16); and (II) disruption of the anastomosis that led to a leakage of the intraluminal content that was sufficient to cause clinical symptoms.

\section{Surgical methods}

All the patients who underwent esophagectomy, which comprised open surgery, video-assisted thoracic surgery (VATS), and robot-assisted thoracic surgery (RATS), underwent cervical anastomosis. During VATS and RATS, the mediastinum was dissected from the diaphragm to the apex of the chest with gentle separation of the fat, nodes, azygos vein, and esophagus. The thorax was then closed 
with the patient in a supine position. The abdomen was explored through a midline incision, and the right gastric and right gastroepiploic arteries were preserved to provide vascular supply to the gastric conduit. Fat and nodes along the celiac trunk and the upper part of the abdominal aorta were removed. The Kocher maneuver procedure was not performed, and the duodenum was not dissociated for extensive retroperitoneal lymphadenectomy during esophagectomy in our institution. The gastric conduit was created by the aid of a linear stapling device, making a conduit 3 to $4 \mathrm{~cm}$ wide along the greater curvature of the stomach. The staple line was oversewn with an additional 3-0 PDS (polydioxanone synthetic) suture. Then, the length of the gastric conduit was measured. To expose the operative field, the patient was placed in a supine position with the neck extended at the base of the skull and the head turned to the right. An oblique incision was made through the skin and muscles on the left side of the neck, and the prepared gastric conduit was gently pulled up with the specimen through the posterior mediastinum and removed through the neck. The anastomosis was created at the posterior wall of the stomach conduit, approximately 2 to $8 \mathrm{~cm}$ from the distal end of the gastric conduit. This distance was determined by the approximate tension of the gastric conduit felt by the surgeon when pulling up the gastric conduit. After completion of the end-to-side handsewn anastomosis, the proximal end of the gastric conduit was removed by stapling $2 \mathrm{~cm}$ from the anastomosis with the same linear stapler. The staple line was oversewn with an additional 3-0 PDS suture (Video 1). The open operation started with a left thoracotomy, after which the esophagus was divided gently from the diaphragm to the apex of the chest. The stomach was explored through an incision in the left diaphragm. After a gastric conduit was created and pulled out into the thorax, the incision in the left diaphragm was sutured, then followed by the same VATS and RATS procedures.

\section{Measurement methods}

Every length was measured by a ruler before or during the operation. The thorax length was defined as the horizontal distance from the xiphoid to the sternal notch, whereas the gastric conduit length started from the pylorus ring and end at the cervical anastomosis. The gastric conduit length was measured externally as follows: (I) the lateral length was measured from the incision level of the conduit to the tip of the conduit; (II) the conduit was fixed, the lateral length was measured from the pylorus ring to the incision level of the conduit, and the original gastric conduit length was equal to the sum of the two aforementioned lengths; and (III) the redundant portion length that starts from the anastomosis and ends at the tip of the conduit was measured. The gastric conduit length is equal to the original gastric conduit length minus the redundant portion length (Figure 1). An index reflecting the tension of the gastric conduit was constructed by dividing the gastric conduit length by the thorax length. For RATS, the robot was removed after the abdominal part of the esophagectomy, and the gastric conduit was created. At this time, the surgeons could measure the gastric conduit length externally.

\section{Statistical analysis}

The chi-square test or Fisher exact test was used to compare categorical parameters. Student's t-test was used to compare continuous variables. A logistic regression in which the anastomotic leakage status was regarded as the dependent variable and the ratio of the gastric conduit length to thorax length, along with other covariates including operation type, operation time, tumor differentiation degree, tumor location, tumor, node, metastasis (TNM) classification and CRP level, were regarded as independent variables, was first conducted to explore if the ratio was a risk indicator. These important clinically variables were entered into regression. Both force-enter selection and backward stepwise selection were performed, and the one with a smaller Akaike information criterion (AIC) value was finally selected. To assist surgeons in making intraoperative recommendations, a cut-off point was needed to divide the patients into distinct risk groups, those who have high or low probabilities of AL occurrence. We conducted a dichotomized analysis comprising an exploratory analysis (graphical diagnostic plots) and an exact search (the minimum $\mathrm{P}$ value approach) to determine the exact cut-off point. A scatter plot and a grouped data plot were drawn in the former stage to help uncover the underlying relationship between the ratio and $\mathrm{AL}$ as well as preliminarily to frame an interval for the potential cut-off point. The scatter plot consisted of all the subjects whose gastric conduit length to thorax length ratio and $\mathrm{AL}$ condition were used as the horizontal and vertical axis values, respectively. All of the ratios were then divided into 10 groups, and the average AL condition within each group was calculated and depicted to form a grouped data plot, from which we identified an interval covering the steepest part. Once the interval was fixed, a contingency 

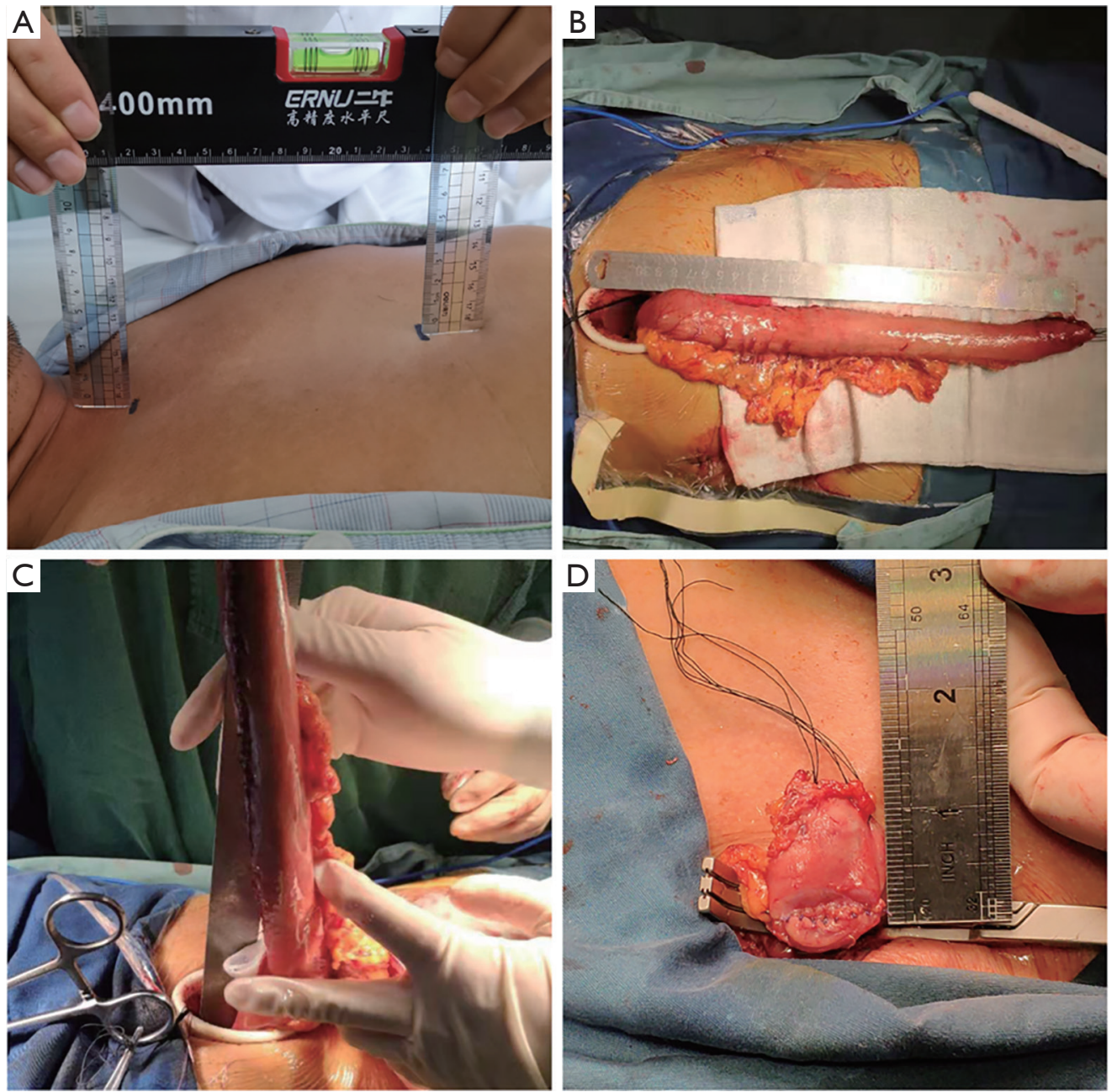

Figure 1 The measurement of gastric tube length and thorax length. (A) The horizontal length of thorax was defined to start at the xiphoid and end at the sternal notch. (B) Measure the lateral length from the incision level to the tip of the conduit. (C) Fix the incision part of the conduit and measure the lateral length from pylorus ring to the incision level. The original gastric conduit length is equal to the sum of the two aforementioned lengths. (D) Measure the length of the resection part that starts from the anastomosis and ends at the tip of the conduit. The gastric conduit length is equal to the original gastric conduit length minus the resection length.

Table 1 The contingency table of every alternative cut-off point

\begin{tabular}{lcc}
\hline & Ratio $\leq C_{0}$ & Ratio $>C_{0}$ \\
\hline With AL & a & b \\
Without AL & $\mathrm{c}$ & $\mathrm{d}$ \\
\hline
\end{tabular}

table was created with every alternative cut-off point, $C_{0}$ (Table 1).

The $C_{0}$ value that produced the minimum $\mathrm{P}$ value or the maximum chi-squared statistic was selected as the cutoff point that best differentiated the AL risk groups. The
Altman correction (17), Bonferroni correction, and Lausen and Schumaker correction (18) were employed to adjust for the inflation in the type I error caused by multiple tests in the search process. The Altman correction provided the following simplified formula to adjust for the $\mathrm{P}$ value:

$$
p_{\text {alt } 10}-a d j=-1.63 p_{\min }\left(1+2.35\left(\operatorname{Ln}\left(p_{\min }\right)\right)\right)
$$

where $p_{\min }$ stands for the observed minimum $\mathrm{P}$ value.

A standard Bonferroni correction was applied by dividing the unadjusted $\mathrm{P}$ value by the number of tests, which was believed to be unappropriated in this situation and updated to a modified version by Lausen and Schumaker. 
Table 2 Patient clinicopathologic characteristics

\begin{tabular}{|c|c|}
\hline Characteristics & $\begin{array}{c}\text { No. of patients } \\
(n, \%) \text { or mean } \pm S D\end{array}$ \\
\hline Age(years) & $64.41 \pm 8.76$ \\
\hline \multicolumn{2}{|l|}{ Gender } \\
\hline Male & $214(78.4)$ \\
\hline Female & $59(21.6)$ \\
\hline $\operatorname{BMI}\left(\mathrm{kg} / \mathrm{m}^{2}\right)$ & $22.94 \pm 3.18$ \\
\hline Diabetes & $20(7.3)$ \\
\hline Hypertension & $62(22.7)$ \\
\hline Smoking history & $138(50.5)$ \\
\hline Drinking history & $89(32.6)$ \\
\hline Pulmonary complication & $24(8.8)$ \\
\hline Cardiovascular complication & $86(31.5)$ \\
\hline \multicolumn{2}{|l|}{ ASA score } \\
\hline I & $187(68.5)$ \\
\hline II & $72(26.4)$ \\
\hline III & $14(5.1)$ \\
\hline \multicolumn{2}{|l|}{ Histological type } \\
\hline SCC & $256(93.8)$ \\
\hline$A C$ & $2(0.7)$ \\
\hline ASC & $2(0.7)$ \\
\hline Others & $13(4.8)$ \\
\hline \multicolumn{2}{|l|}{ Pathologic tumor stage } \\
\hline I & $104(38.1)$ \\
\hline II & $62(22.7)$ \\
\hline III & $99(36.3)$ \\
\hline IV & $8(2.9)$ \\
\hline \multicolumn{2}{|l|}{ Differentiation degree } \\
\hline Well & $71(26.0)$ \\
\hline Moderate & $158(57.9)$ \\
\hline Poor & $44(16.1)$ \\
\hline Preoperative adjuvant therapy & $45(16.5)$ \\
\hline
\end{tabular}

BMI, body mass index; ASA, American Society of Anesthesiologists; SCC, squamous cell carcinoma; AC, adenocarcinoma; ASC, adeno-squamous carcinoma

$$
p_{l s}-a d j=p_{\min }+\sum_{i=1}^{k-1} D\left(\varepsilon_{i}, \varepsilon_{i+1}\right)
$$

where $p_{\text {min }}$ stands for the observed minimum $\mathrm{P}$ value, $k$ stands for the total count of all possible cut-off point alternatives, $\varepsilon_{i}$ is the proportion of the observed values at or below the ith alternative, and the $D()$ function can be explained by:

$D\left(\varepsilon_{i}, \varepsilon_{i+1}\right)=\frac{e\left(-\frac{z^{2}}{2}\right)}{\pi}\left(a\left(\varepsilon_{i}, \varepsilon_{i+1}\right)-\left(\frac{z^{2}}{4}-1\right)\left(\frac{a\left(\varepsilon_{i}, \varepsilon_{i+1}\right)^{3}}{6}\right)\right)$

where $z$ is the $\left(1-\frac{p_{\min }}{2}\right)$ percentile of the standard normal distribution, and the $a()$ function is defined by:

$$
a\left(\varepsilon_{i}, \varepsilon_{i+1}\right)=\sqrt{1-\frac{\varepsilon_{i}\left(1-\varepsilon_{i+1}\right)}{\left(1-\varepsilon_{i}\right) \varepsilon_{i+1}}}
$$

All statistical analyses were implemented with $\mathrm{R}$ (version 3.5.3).

\section{Results}

The clinicopathologic characteristics of the 273 patients are shown in Table 2. The average age of the 273 patients was 64.41 \pm 8.78 . The overall AL incidence was $12.5 \%$ (34/273). Table 3 shows comparisons of the clinicopathologic findings according to the presence or absence of AL. The ratio in patients with AL is significantly lower than that in patients without $\mathrm{AL}(\mathrm{P}<0.001)$. There were no significant differences in the tumor size, tumor location or differentiation degree between patients with AL and patients without AL. A significant difference was found in the operation type between patients with AL and patients without $\mathrm{AL}$ $(\mathrm{P}=0.006)$. The surgical time in patients with $\mathrm{AL}$ was significantly longer than that in patients without AL (0.022). Meanwhile, the TNM stage and the CRP level were both significantly different between patients with $\mathrm{AL}$ and patients without $\mathrm{AL}(\mathrm{P}=0.041$ and $\mathrm{P}<0.001$, respectively).

The correlation between the gastric conduit length and AL is presented in Figure 2. The results of backward stepwise regression and force-enter regression are listed in Table 4 and Table 5, respectively. In the backward stepwise model, which had a smaller AIC value than the forceenter model, covariates other than the ratio, operation type, operation time and CRP were all eliminated. The coefficient (standard error) of the ratio was -7.901 (1.840), which indicated that the larger the ratio was, the lower the tension and the lower risk of $\mathrm{AL}(\mathrm{P}<0.001)$. Compared with operation type 1 (open operation), operation type 2 (VATS) showed a higher AL incidence. A longer operation time, as a result of surgical difficulty, indicated more potential risks. A larger CRP count at POD3 meant more instances of AL. The aforementioned scatter plot and 
Table 3 Clinicopathologic findings according to presence or absence of AL

\begin{tabular}{|c|c|c|c|}
\hline Variables & $\begin{array}{c}A L(-)(n=239) \\
(n, \%)\end{array}$ & $\begin{array}{c}A L(+)(n=34) \\
(n, \%)\end{array}$ & $P$ value \\
\hline Tumor size & & & 0.8 \\
\hline$<3 \mathrm{~cm}$ & $111(46.4)$ & $15(44.1)$ & \\
\hline$\geq 3 \mathrm{~cm}$ & 128 (53.6) & $19(55.9)$ & \\
\hline Tumor location & & & 0.34 \\
\hline Upper & $19(8.0)$ & $1(2.9)$ & \\
\hline Meddle & $140(58.6)$ & $24(70.6)$ & \\
\hline Lower & $80(33.4)$ & $9(26.5)$ & \\
\hline Operation type & & & 0.006 \\
\hline Open operation & $128(53.6)$ & $9(26.5)$ & \\
\hline VATS & 77 (32.2) & $20(58.8)$ & \\
\hline RATS & $34(14.2)$ & $5(14.7)$ & \\
\hline Operation hour & & & 0.022 \\
\hline$<4$ hour & $141(59.0)$ & $13(38.2)$ & \\
\hline$\geq 4$ hour & $98(41.0)$ & $21(61.8)$ & \\
\hline \multicolumn{2}{|c|}{ Differentiation degree } & & 0.88 \\
\hline Well & $61(25.5)$ & $10(29.4)$ & \\
\hline Moderate & 139 (58.2) & $19(55.9)$ & \\
\hline Poor & 39 (16.3) & $5(14.7)$ & \\
\hline TNM stage & & & 0.041 \\
\hline Stage I & $90(37.7)$ & $14(41.2)$ & \\
\hline Stage II & $59(24.7)$ & $3(8.8)$ & \\
\hline Stage III & $85(35.6)$ & $14(41.2)$ & \\
\hline Stage IV & $5(2.0)$ & $3(8.8)$ & \\
\hline Ratio & & & $<0.001$ \\
\hline$<1.79$ & 27 (11.3) & $23(67.6)$ & \\
\hline$\geq 1.79$ & $212(88.7)$ & $11(32.4)$ & \\
\hline CRP & & & $<0.001$ \\
\hline$<73.0$ & 179 (74.9) & $1(2.9)$ & \\
\hline$\geq 73.0$ & $60(25.1)$ & $33(97.1)$ & \\
\hline
\end{tabular}

grouped data plot are illustrated in Figure 3 and Figure 4. The relationship between the ratio and the $\mathrm{AL}$ incidence is nonlinear. The minimum $\mathrm{P}$ value approach was applied to obtain the exact cut-off point value of the ratio (1.79), whereas the unadjusted minimum $\mathrm{P}$ value was $1.245 \times 10^{-14}$
(Table 6). Three adjustments were implemented to correct the inflation of type I error caused by multiple tests (Table 7). The average AL rate below the cut-off point was 17/47 (35.4\%), while the counterpart was 17/225 (7.6\%).

\section{Discussion}

Anastomotic dehiscence remains the main complication in postoperative morbidity and mortality following esophagectomy. Anastomotic leakage can cause some disastrous consequences, such as mediastinitis, sepsis, acute respiratory distress syndrome, or even death (19). The reported AL incidence has consistently decreased in recent years, which further underlines the importance of finding possible contributing factors affecting the occurrence of AL (20-22). Many factors may influence the process of anastomotic healing (14), such as the blood supply $(23,24)$, additional anastomosis between the short gastric vessels and vessels in the neck, the type of gastric conduit (narrow or wide), the method of anastomosis (hand sewing, circular stapler, or triangulating stapling), and the route of anastomosis (retrosternal, posterior mediastinal, or subcutaneous) (19,25-27). Tension in the anastomosis also plays an adverse role in wound healing and is potentially lethal in esophagogastric anastomoses (28).

Although tension is often mentioned in clinical practice, research on the topic of $\mathrm{AL}$ is quite limited because of the obstacles in measuring the tension in esophagogastric anastomoses. We initially considered that the tension may be directly represented by the gastric conduit length; however, the fact that different patients have different thorax lengths can result in a shorter patient with a smaller gastric conduit length having a lower tension than a taller patient with a longer gastric conduit length. Therefore, the thorax length must be taken into consideration to account for the diversity among patients. Originally, we wanted to measure the length of the native esophagus starting from the surface projection of the cardia to the cervical anastomotic site. However, difficulties existed: locating the surface projection of the cardia is difficult; as for the cervical anastomotic site, the position of the incision in the neck is not certain before anastomosis. Therefore, an alternative highly standardized method to measure the length featuring two definite and easy-to-locate endpoints should be employed. First, we took into consideration the length from the spinous process of the 1st thoracic vertebra to the spinous process of the 12th thoracic vertebra; however, this length could be easily affected by scoliosis and kyphosis. Then, the length from 


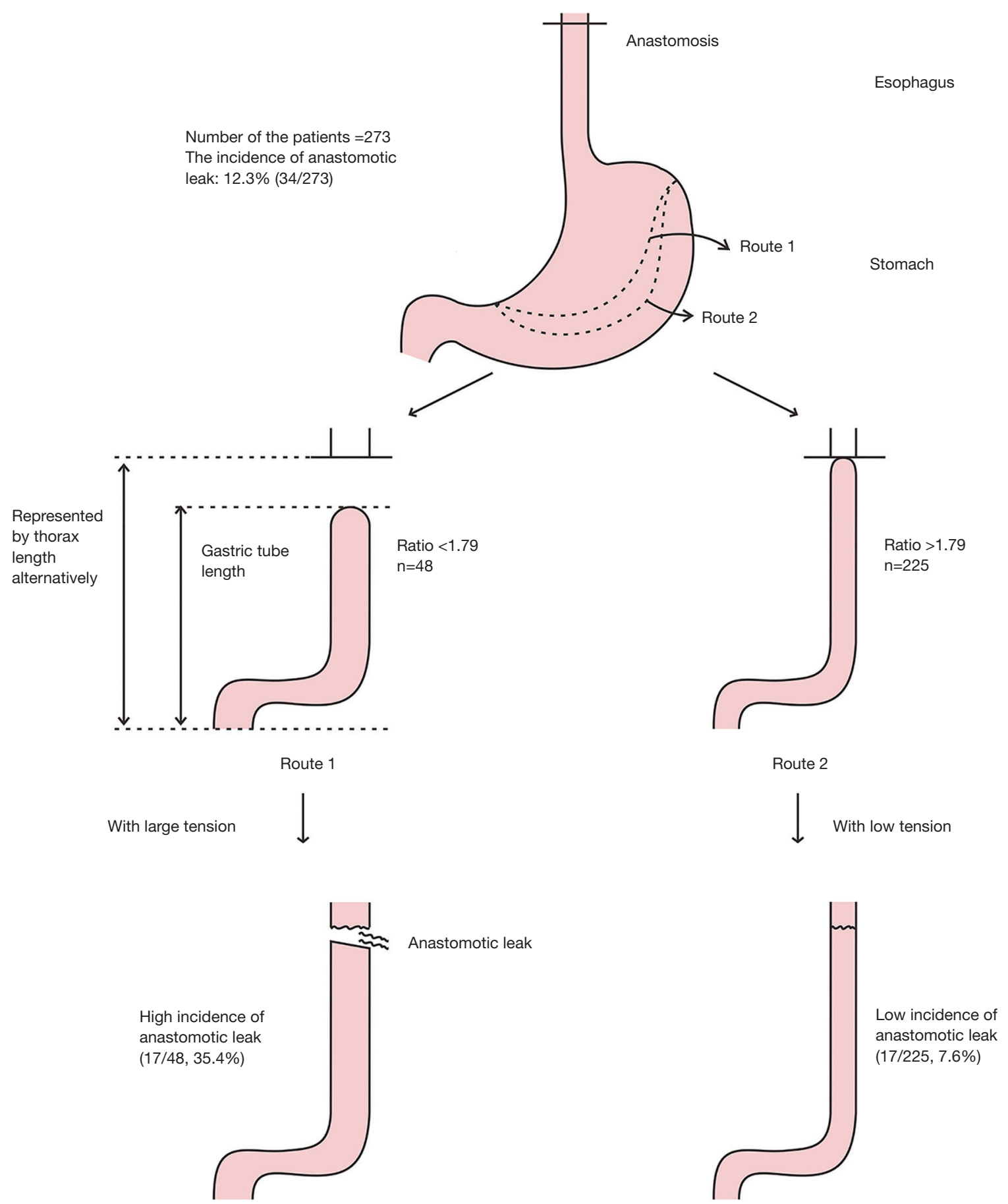

Figure 2 There are different routes by which to trim the stomach during the reconstruction of the gastric tract, which influence the gastric conduit length. The tension in the anastomosis can then be represented alternatively by the ratio of the gastric conduit length to the thorax length. The bigger the ratio is, the smaller the risk of AL will be. Therefore, surgeons can properly increase the gastric conduit length according to the patient's thorax length during the reconstruction of the gastric tract, reducing the tension in the anastomosis and finally leading to a decrease in AL incidence. 
Table 4 Backward stepwise selection result (AIC: 99.26)

\begin{tabular}{lccc}
\hline Covariate name & Coefficient & Standard error & P value \\
\hline Ratio & -7.751 & 1.840 & $<0.001$ \\
Operative type & & & \\
Open operation & Reference & & \\
VATS & 2.052 & 1.091 & 0.060 \\
RATS & 0.054 & 0.681 & 0.936 \\
Operative hour & 1.12 & 0.374 & 0.003 \\
CRP & 0.022 & 0.004 & $<0.001$ \\
\hline
\end{tabular}

AIC, Akaike information criterion; VATS, video-assisted thoracic surgery; RATS, robot-assisted thoracic surgery; CRP, C-reactive protein.

Table 5 Force-enter selection result (AIC: 106.76)

\begin{tabular}{|c|c|c|c|}
\hline Covariate name & Coefficient & Standard error & $P$ value \\
\hline Ratio & -7.939 & 2.019 & $<0.001$ \\
\hline \multicolumn{4}{|l|}{ Operative type } \\
\hline Open operation & Reference & & \\
\hline VATS & 2.337 & 1.177 & 0.047 \\
\hline RATS & 0.278 & 0.763 & 0.725 \\
\hline Operative hour & 1.233 & 0.424 & 0.004 \\
\hline \multicolumn{4}{|c|}{ Differentiation degree } \\
\hline Well & Reference & & \\
\hline Moderate & 1.08 & 0.857 & 0.208 \\
\hline Poor & 1.073 & 1.040 & 0.302 \\
\hline \multicolumn{4}{|l|}{ TNM stage } \\
\hline I & Reference & & \\
\hline II & -0.422 & 0.942 & 0.654 \\
\hline III & -0.172 & 0.721 & 0.812 \\
\hline IV & 2.201 & 1.228 & 0.073 \\
\hline \multicolumn{4}{|l|}{ Tumor location } \\
\hline Upper & Reference & & \\
\hline Middle & -1.015 & 1.343 & 0.45 \\
\hline Lower & -1.805 & 1.486 & 0.225 \\
\hline CRP & 0.025 & 0.005 & $<0.001$ \\
\hline
\end{tabular}

AIC, Akaike information criterion; VATS, video-assisted thoracic surgery; RATS, robot-assisted thoracic surgery; TNM, tumor, node, metastasis; CRP, C-reactive protein. the xiphoid to the sternal notch was considered. However, this measure was easily affected by sex and BMI (body mass index) and could not precisely represent the length of the native esophageal bed in the posterior mediastinum. Finally, we chose the horizontal length of the thorax, which was defined as the distance between the start at the xiphoid and the end of the sternal notch; this measure correlates to the length of the native esophageal bed more precisely.

In our study, the thorax length and the gastric conduit length were measured before and during the operation, respectively. We then constructed an index that reflected the tension of the esophagogastric anastomoses by dividing the gastric conduit length by the thorax length. Therefore, it is rather clear that a larger ratio implies less tension. Given general knowledge that higher tension after anastomosis can lead to a higher AL incidence (28), it is likely that controlling the ratio to be within an appropriate range during surgery can reduce the incidence of AL.

During the surgeries, gastric conduits were positioned in a uniform manner in the posterior mediastinum, and in this study, anastomoses in the neck were created manually due to the high standardization of this technique as well as to avoid the reported high failure rate of stapled anastomoses attempted in the neck (29). Some studies have suggested that cervical anastomosis in esophagectomy can reduce the recurrence of esophageal cancer. The clinical outcomes of thoracic anastomotic leakage are less favorable than those of cervical anastomotic leakage, and the management of patients with cervical anastomotic leakage is much easier than that for patients with thoracic anastomotic leakage. Therefore, in our institution, almost all patients with esophageal cancer underwent cervical anastomosis.

Our study demonstrates four significant risk factors for AL: operation time, operation type, POD3 CRP level, and the ratio of gastric conduit length to thorax length. The operation time, which may be influenced by pleural adhesions, large tumor size, bleeding, or other reasons, can determine the difficulty of the surgery to some extent. Long-term isolation of the gastric conduit can reduce the blood supply after anastomosis; moreover, patients may produce a more serious postoperative stress response after the pleural adhesions are divided or after bleeding occurs during surgery. The results showed that open operations yielded a lower AL incidence compared with VATs, which may be caused by the collection bias since the number of patients included in this study was limited. Therefore, 


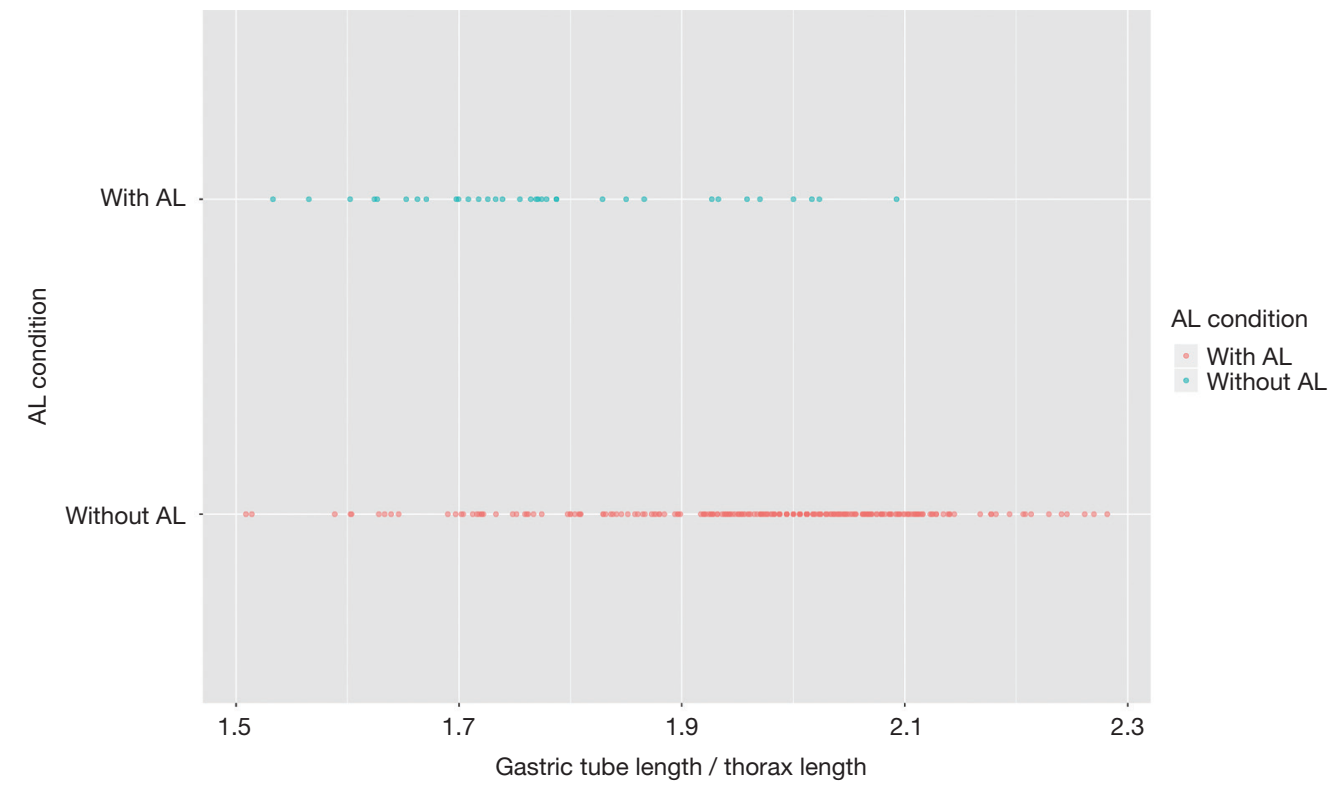

Figure 3 Scatter plot of ratio in an AL condition. Figure 3 shows the ratios (the gastric conduit length divided by the thorax length) and the $\mathrm{AL}$ (anastomotic leak) conditions (with or without AL) of all 273 subjects. Each point represents a specific subject with the $x$-axis standing for the ratio and the $y$-axis standing for the AL condition. Subjects who suffer from AL tend to have a smaller ratio, whereas those without AL feature a larger ratio, which is indicated by the transparency of the points.

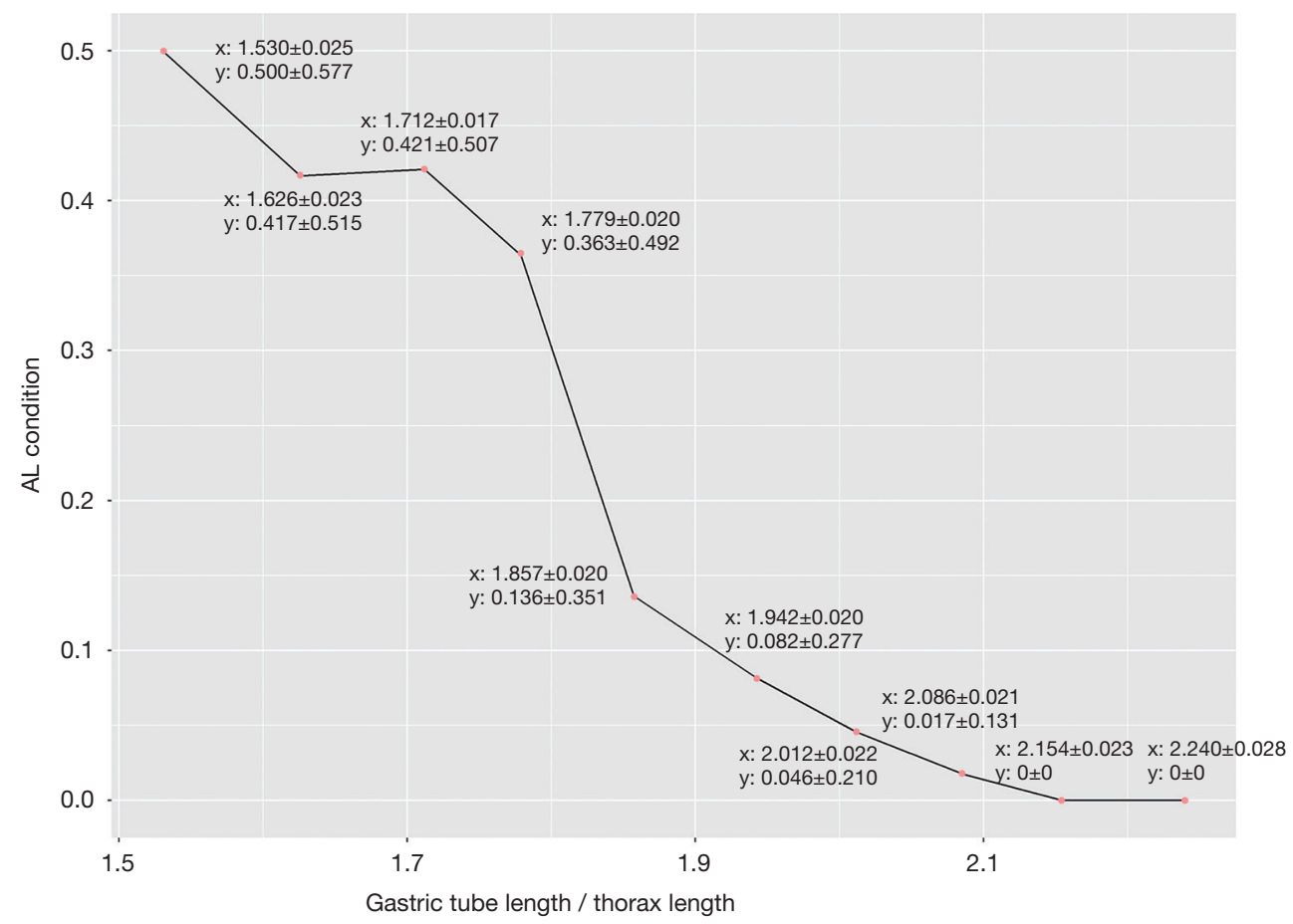

Figure 4 Grouped data plot of the ratio in an AL condition. Figure 4 is a preliminarily smoothed version on the base of the scatter plot in Figure 2. All 273 points are divided equally into 10 bins, according to the ratio. Within each group, the arithmetic mean and the standard deviation of the ratio are then calculated and labeled (e.g., x: $1.530 \pm 0.025$ ), as well as those of the AL condition (e.g., y: 0.500 \pm 0.577 ). The steepest sector can be found on the line chart to form an interval where the potential dichotomization point will be sought exactly, later. 
Table 6 Minimum P value approach search result

\begin{tabular}{|c|c|c|}
\hline Alternative ratio & Chi-squares & $P$ value \\
\hline 1.74 & 30.912 & $2.700 \times 10^{-8}$ \\
\hline 1.75 & 29.444 & $5.757 \times 10^{-8}$ \\
\hline 1.76 & 31.078 & $2.479 \times 10^{-8}$ \\
\hline 1.77 & 35.904 & $2.073 \times 10^{-9}$ \\
\hline 1.78 & 48.892 & $2.704 \times 10^{-12}$ \\
\hline 1.79 & 59.466 & $1.245 \times 10^{-14}$ \\
\hline 1.80 & 54.283 & $1.736 \times 10^{-13}$ \\
\hline 1.81 & 48.238 & $3.776 \times 10^{-12}$ \\
\hline 1.82 & 48.238 & $3.776 \times 10^{-12}$ \\
\hline 1.83 & 50.329 & $1.301 \times 10^{-12}$ \\
\hline 1.84 & 46.376 & $9.760 \times 10^{-12}$ \\
\hline 1.85 & 48.576 & $3.178 \times 10^{-12}$ \\
\hline 1.86 & 46.161 & $1.089 \times 10^{-11}$ \\
\hline 1.87 & 47.292 & $6.117 \times 10^{-12}$ \\
\hline 1.88 & 41.997 & $9.141 \times 10^{-11}$ \\
\hline 1.89 & 41.021 & $1.506 \times 10^{-10}$ \\
\hline 1.90 & 37.363 & $9.807 \times 10^{-10}$ \\
\hline
\end{tabular}

Table 7 Three corrections for the unadjusted $\mathrm{P}$ value

\begin{tabular}{lccc}
\hline Unadjusted & Altman & $\begin{array}{c}\text { Standard } \\
\text { Bonferroni }\end{array}$ & $\begin{array}{c}\text { Lausen \& } \\
\text { Schumaker }\end{array}$ \\
\hline $1.245 \times 10^{-14}$ & $1.506 \times 10^{-12}$ & $2.116 \times 10^{-13}$ & $1.359 \times 10^{-13}$ \\
\hline
\end{tabular}

we propose further studies to explore the effect of these three surgical methods on postoperative complications. Postoperatively elevated CRP levels have been reported to be predictive of postoperative systemic inflammation reactions and complications, and this factor has already been used as a predictor of AL (30). In our study, a similar conclusion was drawn: higher POD3 CRP levels tends to enhance the risk of AL.

We further explored the influence of the gastric conduit length to thorax length ratio on tension in anastomosis. According to our results, a smaller ratio leads to a higher incidence of AL, and a steep drop can be seen in Figure 4, from 1.74 to 1.90 , which indicates that when the ratio falls within this range, the occurrence of $\mathrm{AL}$ will change significantly. There are three potential explanations for this phenomenon: (I) tension exists intrinsically and leads to the occurrence of $\mathrm{AL}$; (II) when the ratio falls below some critical value within this interval, the tension in the anastomosis is more likely to become higher than the traction in the suture, which can lead to a breakage or a loosening of the suture and then cause an early AL; and (III) even if an early AL does not occur, long-term effects still exist: both potential gaps in the anastomosis and constant tension in the gastric conduits that squeeze the short gastric vessels can impair healing, causing an occurrence of a late AL. Therefore, a cut-off value for the ratio should be found as a guide for surgeons to reduce the incidence of AL.

The accurate cut-off value search gave an optimal dichotomization point of 1.79 , which means that the AL incidence might decrease if the surgeon ensures that the ratio higher than 1.79 . The ratio is influenced by both the gastric conduit length and the thorax length; however, the latter is always fixed in a particular patient, which means that we should control the former to increase the ratio. Moreover, the gastric conduit length was determined by both the route of tailoring the stomach and the redundant portion length. Given that the distance of the reconstruction of the alimentary tract for cervical anastomosis is long, surgeons should choose an optimal route that could allow the gastric conduit to be long enough for reconstruction, while ensuring that the diameter of the gastric conduit is not too small. The diameters of the gastric conduits in our study were between 3 and 4 centimeters. If the stomach size was not large enough for reconstruction based on the experience of the surgeons, the width of the gastric conduit was reduced appropriately to increase the length. Measurements should be taken after creating the initial gastric conduit, and then the measured length should be compared with the value of the thorax length multiplied by 1.79 . If the initial gastric conduit length is smaller, surgeons should anastomose to the most distal point of the conduit, since controlling the ratio is more important than removing the redundant portion of the proximal conduit, according to our results. Otherwise, surgeons should anastomose to a certain position to remove as much of the redundant portion that is most ischemic as possible. In this case, the redundant portion length should be equal to the initial gastric conduit length minus the value of the thorax length multiplied by 1.79 .

\section{Limitation}

The main limitations are as follows: i. no background 
data supporting that the ratio is correlated to anastomotic tension were found, and we suggest that further studies should provide more information on this topic; and ii. only the end-to-side hand-sewn anastomosis was included in our study, and therefore, we did not address the different techniques of other surgeons regarding where the anastomosis is made on the conduit.

\section{Conclusions}

The ratio of the gastric conduit length to the thorax length can reflect the tension in the anastomosis. An optimal dichotomization point of 1.79 was determined to be a guide in clinical practice. This study proposes that surgeons can properly increase the gastric conduit length according to the cut-off point and the patient's thorax length during the reconstruction of the esophageal tract to reduce tension in the anastomosis and that this approach can finally lead to a reduction in the incidence of $\mathrm{AL}$.

\section{Acknowledgments}

We would like to thank Dr. Gretta $\mathrm{Wu}$ for her help in polishing the language of our paper.

Funding: This work is supported by the National Natural Science Foundation of China (NO. 81172032) and the Natural Science Foundation of Jiangsu Province (BK20181239).

\section{Footnote}

Reporting Checklist: The authors have completed the MDAR reporting checklist. Available at http://dx.doi.org/10.21037/ atm-20-6082

Data Sharing Statement: Available at http://dx.doi. org/10.21037/atm-20-6082

Peer Review File: Available at http://dx.doi.org/10.21037/ atm-20-6082

Conflicts of Interest: All authors have completed the ICMJE uniform disclosure form (available at http://dx.doi. org/10.21037/atm-20-6082). The authors have no conflicts of interest to declare.

Ethical Statement: The authors are accountable for all aspects of the work in ensuring that questions related to the accuracy or integrity of any part of the work are appropriately investigated and resolved. The study was conducted in accordance with the Declaration of Helsinki (as revised in 2013) and the Harmonized Tripartite Guideline for Good Clinical Practice from the International Conference on Harmonization. This study was approved by the Institutional Review Board of the Jinling Hospital (approval number 2018NZKY-027-54) and individual consent for this retrospective analysis was waived. The study outcomes will not affect the future patient management. This study is based on data retrieved from a hospital medical record system. All personal data have been protected and secured according to current national and international laws.

Open Access Statement: This is an Open Access article distributed in accordance with the Creative Commons Attribution-NonCommercial-NoDerivs 4.0 International License (CC BY-NC-ND 4.0), which permits the noncommercial replication and distribution of the article with the strict proviso that no changes or edits are made and the original work is properly cited (including links to both the formal publication through the relevant DOI and the license). See: https://creativecommons.org/licenses/by-nc-nd/4.0/.

\section{References}

1. Alanezi K, Urschel JD. Mortality secondary to esophageal anastomotic leak. Ann Thorac Cardiovasc Surg 2004;10:71-5.

2. Udagawa H, Akiyama H. Surgical treatment of esophageal cancer: Tokyo experience of the three-field technique. Dis Esophagus 2001;14:110-4.

3. Ando N, Ozawa S, Kitagawa Y, et al. Improvement in the results of surgical treatment of advanced squamous esophageal carcinoma during 15 consecutive years. Ann Surg 2000;232:225-32.

4. Korst RJ, Port JL, Lee PC, et al. Intrathoracic manifestations of cervical anastomotic leaks after transthoracic esophagectomy for carcinoma. Ann Thorac Surg 2005;80:1185-90.

5. Atkins BZ, Shah AS, Hutcheson KA, et al. Reducing hospital morbidity and mortality following esophagectomy. Ann Thorac Surg 2004;78:1170-6; discussion 1176.

6. Sarela AI, Tolan DJ, Harris K, et al. Anastomotic leakage after esophagectomy for cancer: a mortality-free experience. J Am Coll Surg 2008;206:516-23.

7. Chen $\mathrm{C}, \mathrm{Yu} \mathrm{Z}$, Jin Q, et al. Clinical features and risk 
factors of anastomotic leakage after radical esophagectomy. Zhonghua Wai Ke Za Zhi 2015;53:518-21.

8. Rutegard M, Lagergren P, Rouvelas I, et al. Intrathoracic anastomotic leakage and mortality after esophageal cancer resection: a population-based study. Ann Surg Oncol 2012;19:99-103.

9. Whooley BP, Law S, Alexandrou A, et al. Critical appraisal of the significance of intrathoracic anastomotic leakage after esophagectomy for cancer. Am J Surg 2001;181:198-203.

10. Messager M, Warlaumont M, Renaud F, et al. Recent improvements in the management of esophageal anastomotic leak after surgery for cancer. Eur J Surg Oncol 2017;43:258-69.

11. Davies N. Surgeon volumes in oesophagogastric and hepatopancreatobiliary resectional surgery (Br J Surg 2011; 98: 891-893). Br J Surg 2011;98:1496; author reply 1497.

12. Zhang SS, Yang H, Luo KJ, et al. The impact of body mass index on complication and survival in resected oesophageal cancer: a clinical-based cohort and meta-analysis. Br J Cancer 2013;109:2894-903.

13. van Rossum PSN, Haverkamp L, Verkooijen HM, et al. Calcification of arteries supplying the gastric tube: a new risk factor for anastomotic leakage after esophageal surgery. Radiology 2015;274:124-32.

14. Shimada Y, Okumura T, Nagata T, et al. Usefulness of blood supply visualization by indocyanine green fluorescence for reconstruction during esophagectomy. Esophagus 2011;8:259-66.

15. Rino Y, Yukawa N, Sato T, et al. Visualization of blood supply route to the reconstructed stomach by indocyanine green fluorescence imaging during esophagectomy. BMC Med Imaging 2014;14:18.

16. Okamura A, Watanabe M, Imamura Y, et al. Preoperative Glycosylated Hemoglobin Levels Predict Anastomotic Leak After Esophagectomy with Cervical Esophagogastric Anastomosis. World J Surg 2017;41:200-7.

17. Altman DG, Lausen B, Sauerbrei W. Dangers of Using $\backslash$ "Optimal\" Cutpoints in the Evaluation of Prognostic Factors. Journal of the National Cancer Institute 1994;86:829-35.

18. Lausen B, Schumacher M. Evaluating the e!ect of optimized cuto! values in the assessment of prognostic factors. Computational Statistics \& Data Analysis 1996;21:307-26.
19. Michelet P, D'Journo XB, Roch A, et al. Perioperative risk factors for anastomotic leakage after esophagectomy: influence of thoracic epidural analgesia. Chest 2005;128:3461-6.

20. Pickleman J, Watson W, Cunningham J, et al. The failed gastrointestinal anastomosis: an inevitable catastrophe? J Am Coll Surg 1999;188:473-82.

21. Bardini R, Asolati M, Ruol A, et al. Anastomosis. World J Surg 1994;18:373-8.

22. Voros A, Ender F, Jakkel T, et al. Esophageal anastomosis-based on the experience with 1460 operations. Magy Seb 2001;54:132-7.

23. Miyazaki T, Kuwano H, Kato H, et al. Predictive value of blood flow in the gastric tube in anastomotic insufficiency after thoracic esophagectomy. World J Surg 2002;26:1319-23.

24. Ikeda Y, Niimi M, Kan S, et al. Clinical significance of tissue blood flow during esophagectomy by laser Doppler flowmetry. J Thorac Cardiovasc Surg 2001;122:1101-6.

25. Toh Y, Sakaguchi Y, Ikeda O, et al. The triangulating stapling technique for cervical esophagogastric anastomosis after esophagectomy. Surg Today 2009;39:201-6.

26. Lerut T, Coosemans W, Decker G, et al. Anastomotic complications after esophagectomy. Dig Surg 2002;19:92-8.

27. Pierie JP, de Graaf PW, van Vroonhoven TJ, et al. The vascularization of a gastric tube as a substitute for the esophagus is affected by its diameter. Dis Esophagus 1998;11:231-5.

28. Rahamim J, Cham CW. Oesophagogastrectomy for carcinoma of the oesophagus and cardia. Br J Surg 1993;80:1305-9.

29. Chasseray VM, Kiroff GK, Buard JL, et al. Cervical or thoracic anastomosis for esophagectomy for carcinoma. Surg Gynecol Obstet 1989;169:55-62.

30. Guillem P, Triboulet JP. Elevated serum levels of C-reactive protein are indicative of a poor prognosis in patients with esophageal cancer. Dis Esophagus 2005;18:146-50.

Cite this article as: $\mathrm{Li} \mathrm{XK}$, Hua TT, Zhang $\mathrm{C}, \mathrm{Xu} \mathrm{Y}$, Wu WJ, Zheng C, Wang GM, Qiang Y, Cong ZZ, Yi J, Shen Y. The ratio of gastric tube length to thorax length: a vital factor affecting leak after esophageal cervical anastomosis. Ann Transl Med 2021;9(6):458. doi: 10.21037/atm-20-6082 